\title{
Spatio-temporal characterization of the electric field of breathers in an optical fiber
}

\author{
Corentin Naveau ${ }^{1}$, Pascal Szriftgiser ${ }^{1}$, Alexandre Kudlinski ${ }^{1}$, Matteo Conforti ${ }^{1}$, Stefano Trillo $^{2}$, \\ and Arnaud Mussot ${ }^{1}$ \\ 1. Univ. Lille, CNRS, UMR 8523-PhLAM - Physique des Lasers Atomes et Molécules, F-59000 Lille, France \\ 2. Department of Engineering, University of Ferrara, Via Saragat 1, 44122 Ferrara, Italy
}

Full experimental characterization of short pulses (with discrete line spectra) of the electric field (phase+intensity) in optical fibers is of high interest to get a detailed description of a large variety of nonlinear phenomena governed by the general nonlinear Schrödinger equation such as rogue wave formation or frequency comb generation. Recently, new techniques allowing real-time full-field characterization has been shown in the context of rogue waves [1] and laser dynamics [2]. However, experimental methods were limited so far to localized characterization of the field.
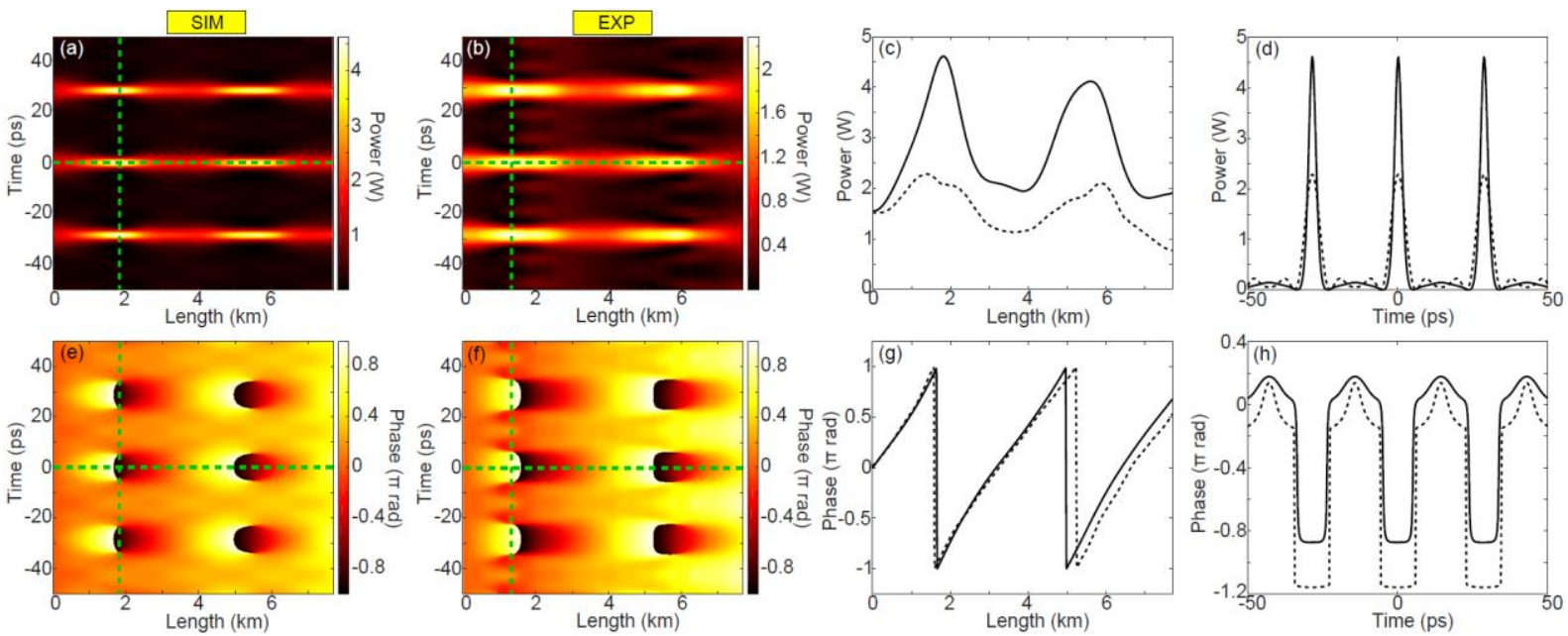

Fig. 1 Spatio-temporal evolution of power ((a) numerical simulations, (b) experiments) and phase ((e) numerical simulations, (f) experiments). $(\mathrm{c}, \mathrm{g})$ Longitudinal and temporal $(\mathrm{d}, \mathrm{h})$ evolutions of power and phase at the first maximum compression point (along the green dashed lines). Black dashed lines correspond to experiments and solid ones to numerical simulations with the full spectrum.

In this work we report the first non-invasive technique allowing characterization of the full-field longitudinal evolution in an optical fiber of breathers in the picosecond scale by mean of a heterodyne optical time domain reflectometer [3]. This system allows us to perform distributed measurements along the fiber of the intensity and phase of the five main spectral components of the pulses (the pump, the first and second order sidebands). Then, via inverse Fourier transform, we calculate the longitudinal evolution of the field in the time domain as displayed in Fig. 1(b) and (f). We observe two maximum compressions (at around $1.5 \mathrm{~km}$ and $6 \mathrm{~km}$ ) which occurrence can be linked to the Fermi-Pasta-Ulam process [4]. The comparison with numerics (Fig. 1(a) and (e)) obtained by integrating the nonlinear Schrödinger equation shows a good agreement. We also plot the longitudinal and temporal evolutions of the field along the first maximum compression point in Fig. 1(c,d,g,h) and get a pretty good agreement too. Discrepancies between experimental traces (dashed lines) and numerical ones (solid lines) are mainly explained by the use of a truncated spectrum in experiments (five waves) compared to the full spectrum in simulations (dozens of waves) to calculate the inverse Fourier transform [3]. Moreover, by changing the initial input modulation phase, we were able to observe two maximum compression points but with a $\pi$ phase shift (not shown here), which is the first observation of the symmetry breaking nature of the FPU process in the time domain [3].

In conclusion, our original experimental setup allowed the full-field spatio-temporal characterization of breather dynamics along an optical fiber in the context of Fermi-Pasta-Ulam recurrences on a picosecond scale.

\section{References}

[1] A. Tikan, S. Bielawski, C. Szwaj, S. Randoux, and P. Suret, Single-shot measurement of phase and amplitude by using a heterodyne timelens system and ultrafast digital time-holography, Nat. Phot. 12, 228-234 (2018).

[2] P. Ryczkowski, M. Närhi, C. Billet, J.-M. Merolla, G. Genty, and J. M. Dudley, Real-time full-field characterization of transient dissipative soliton dynamics in a mode-locked laser, Nat.Phot. 12, 221-227 (2018).

[3] C. Naveau, P. Szriftgiser, A. Kudlinski, M. Conforti, S. Trillo ans A. Mussot, "Full-Field characterization of breather dynamics along the whole length of an optical fiber," Opt. Lett. (to appear in 2019).

[4] G. Van Simaeys, Ph. Emplit, M. Halterman, Experimental Demonstration of the Fermi-Pasta-Ulam Recurrence in a Modulationally Unstable Optical Wave, Phys. Rev. Lett. 87, 033902 (2001). 